\title{
A104 IL-23 INHIBITS IL-22 AND AHR SIGNALLING AND WITH IL-1 $\beta$ REGULATES THE HUMAN PERIPHERAL TH17/TH22 BALANCE
}

Ferry Cornelissen, Jan Piet van Hamburg, Nadine Davelaar, Patrick S Asmawidjaja, Adriana MC Mus, Erik Lubberts Departments of Rheumatology and Immunology, Erasmus Medical Center Rotterdam, Rotterdam, The Netherlands

10.1136/ard.2010.148981.7

Background and objective Human peripheral memory $\left(\mathrm{CD} 45 \mathrm{RO}^{+}\right)$Th17 cells express the chemokine receptor CCR6 and these cells have been found in elevated numbers in chronic inflammatory diseases including rheumatoid arthritis (RA) and have recently been shown to participate in the chronicity of synovial inflammation. Additionally, a minor population

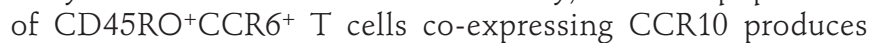
interleukin (IL)-22, little IL-17A and no IFN $\gamma$ and has therefore been named Th22. Furthermore, it seemed that the aryl hydrocarbon receptor (AhR) positively controls IL-22 expression. Although the direct role of these CCR ${ }^{+}$CCR $10^{+}$Th2 22 cells is still unclear in RA, CCR10 is involved in skin-homing so Th22 cells might have a direct contribution to skin pathology and perhaps in other organ-specific autoimmune disorders as well. In fact, IL-22 was found to be involved in arthritis and in skin pathology.

Here, the authors investigated the role of the Th17-polarising cytokines IL-23 and IL-1 $\beta$ in the regulation of human peripheral Th17 and Th22 cells.

Materials and methods Human peripheral memory Th17 $\left(\mathrm{CD} 4{ }^{+} \mathrm{CD} 45 \mathrm{RO}^{+} \mathrm{CCR} 6^{+}\right)$and Th22 $\left(\mathrm{CD} 4{ }^{+} \mathrm{CD} 45 \mathrm{RO}{ }^{+} \mathrm{CCR} 6{ }^{+} \mathrm{CCR} 10^{+}\right)$cells were FACS-sorted and activated with anti-CD3 plus anti-CD28 for 3 days in the presence of IL-23, IL-13, 6-formylindolo[3,2-b]carbazole (FICZ), or combinations. Post culture, cells were analysed by flow cytometry for intracellular IL-17A, IL-22 and IFN $\gamma$ expression and by quantitative real-time PCR. Cytokine secretion was measured by ELISA.

Results Here, the authors show the potential of IL-23 and IL- $1 \beta$ in the regulation of IL-17A and IL-22 by human memory CCR6 $6^{+}$Th17 and CCR6 $6^{+}$CCR $10^{+}$Th22 cells. Whereas IL-1 $\beta$ strongly enhanced the proportion of CCR $6^{+}$Th17 cells and IL-17A secretion, IL-23 significantly suppressed IL-22 production. When combined, IL-23 and IL-1 $\beta$ regulated the balance of IL-17A ${ }^{+}$and IL-22+ ${ }^{+}$CRR ${ }^{+}$cells in favour of IL-17A. The regulation of IL-17A and IL-22 by IL-23 and IL- $1 \beta$ went further than CCR6 ${ }^{+}$Th17 cells since in CCR ${ }^{+}$CCR $10^{+}$Th 22 cells, besides suppressing IL-22, FICZ-induced CYP1A1 expression was clearly inhibited indicating that IL-23 hinders the AhRsignalling. Conversely, IL-1 $\beta$ induced high levels of IL-17A in these CCR $10^{+}$Th22 cells. Remarkably, CCR $6^{+}$CCR $10^{+}$Th22 cells expressed lower levels of IL-23 receptor transcripts (but similar levels of IL-1R1) than their CCR ${ }^{+}$CCR10- counterparts suggesting that Th22 cells are less responsive to IL-23 stimulation than Th17 cells.

Conclusion These data show that IL-23 and IL-1 $\beta$ co-operate in the regulation of IL-17A and IL-22 in peripheral human memory Th17 and Th22 cells in favour of Th17. These data add new insight in the role of IL-23 and IL- $1 \beta$ in the regulation of the potential pathogenic Th17 and Th22 subsets. 